\title{
Pluripotent stem cell-derived CAR-macrophage cells with antigen-dependent anti-cancer cell functions
}

\author{
Li Zhang ${ }^{1,2+}{ }^{1,}$ Lin Tian ${ }^{1,2+}$, Xiaoyang Dai ${ }^{3 \dagger}$, Hua Yu ${ }^{1,2+}$, Jiajia Wang ${ }^{3 \dagger}$, Anhua Lei ${ }^{1,2}$, Mengmeng Zhu ${ }^{1,2}$,

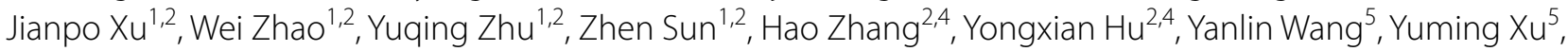 \\ George M. Church ${ }^{6}$, He Huang ${ }^{2,4,7^{*}}$, Qinjie Weng ${ }^{3^{*}}$ and Jin Zhang ${ }^{1,2,7^{*}}$
}

\begin{abstract}
The Chimera antigen receptor (CAR)-T cell therapy has gained great success in the clinic. However, there are still major challenges for its wider applications in a variety of cancer types including lack of effectiveness due to the highly complex tumor microenvironment, and the forbiddingly high cost due to the personalized manufacturing procedures. In order to overcome these hurdles, numerous efforts have been spent focusing on optimizing Chimera antigen receptors, engineering and improving T cell capacity, exploiting features of subsets of T cell or NK cells, or making off-theshelf universal cells. Here, we developed induced pluripotent stem cells (iPSCs)-derived, CAR-expressing macrophage cells (CAR-iMac). CAR expression confers antigen-dependent macrophage functions such as expression and secretion of cytokines, polarization toward the pro-inflammatory/anti-tumor state, enhanced phagocytosis of tumor cells, and in vivo anticancer cell activity. This technology platform for the first time provides an unlimited source of iPSC-derived engineered CAR-macrophage cells which could be utilized to eliminate cancer cells.
\end{abstract}

Keywords: Chimera antigen receptor (CAR), Induced pluripotent stem cells (iPSC)-derived macrophage cells (iMac), Antigen-dependent activation, Anti-cancer cell functions

\section{To the Editor,}

Recently, CAR-iPSC-differentiated CAR-expressing $\mathrm{T}$ cells and NK cells have been reported to have potent cytotoxic activity against cancer cells, and they represent a new family of engineered stem cell-derived immune

\footnotetext{
*Correspondence: hehuangyu@126.com; wengqinjie@zju.edu.cn; zhgene@zju.edu.cn

${ }^{+}$Li Zhang, Lin Tian, Xiaoyang Dai, Hua Yu and Jiajia Wang have contributed equally to this work

${ }^{1}$ Center for Stem Cell and Regenerative Medicine, Department of Basic Medical Sciences, and The First Affiliated Hospital, Zhejiang University School of Medicine, Hangzhou, 310058, China

${ }^{2}$ Institute of Hematology, Zhejiang University, Hangzhou 310058, China

${ }^{3}$ Zhejiang Province Key Laboratory of Anti-Cancer Drug Research, Center for Drug Safety Evaluation and Research, Institute of Pharmacology and Toxicology, College of Pharmaceutical Sciences, Zhejiang University, Hangzhou 310058, China

Full list of author information is available at the end of the article
}

cells for CAR therapies [1, 2]. Myeloid cells such as macrophages have been utilized as a type of effector cells to combat cancer cells by means of their phagocytosis function [3, 4]. However, immortalized macrophage cell lines are not applicable to clinical settings, and bone marrow or PBMC-derived primary macrophages are not efficiently engineered, thus leaving iPSC-derived macrophage cells as a great source for myeloid cell-based immunotherapy. Upon challenge with antigen-expressing cancer cells, CAR-expressing iPSC-induced macrophage (CAR-iMac) cells showed antigen-dependent macrophage functions. Expression of a CAR targeting tumorassociated antigen conferred CAR-iMac cells in vitro and in vivo anti-tumor effects.

First, we started from deriving iPSCs from peripheral blood mononuclear cells (PBMC) of a healthy donor with original author(s) and the source, provide a link to the Creative Commons licence, and indicate if changes were made. The images or other third party material in this article are included in the article's Creative Commons licence, unless indicated otherwise in a credit line to the material. If material is not included in the article's Creative Commons licence and your intended use is not permitted by statutory regulation or exceeds the permitted use, you will need to obtain permission directly from the copyright holder. To view a copy of this licence, visit http://creativecommons.org/licenses/by/4.0/. The Creative Commons Public Domain Dedication waiver (http://creativeco mmons.org/publicdomain/zero/1.0/) applies to the data made available in this article, unless otherwise stated in a credit line to the data. 
non-integrating episomal vectors encoding reprogramming factors (Fig. 1a), and isolated single iPSC clones (Additional file 1: Fig. S1). The materials and methods are shown in detail in the Additional file 2. Then, we compared different CD19 CARs and chose the conventional CAR to introduce into the iPSCs by lentiviral transduction (Fig. 1a and Additional file 1: Fig. S2a-g). Then, we established a protocol of myeloid/macrophage differentiation to induce CAR-iPSCs toward myeloid cell lineages
(Additional file 1: Fig. S2h). Differentiated cells showed typical macrophage marker gene expression (Fig. 1b, Additional file 1: Fig. S4b-i). The products can be collected from 20 to 30 day for multiple times to allow further expansion to have a final yield of above 50-fold of the starting iPSCs (Additional file 1: Fig. S3a), with high purity indicated by $\sim 100 \%$ of CD11b and CD14 expression at later days (Fig. 1b). Key macrophage marker genes were induced, whereas pluripotent marker genes

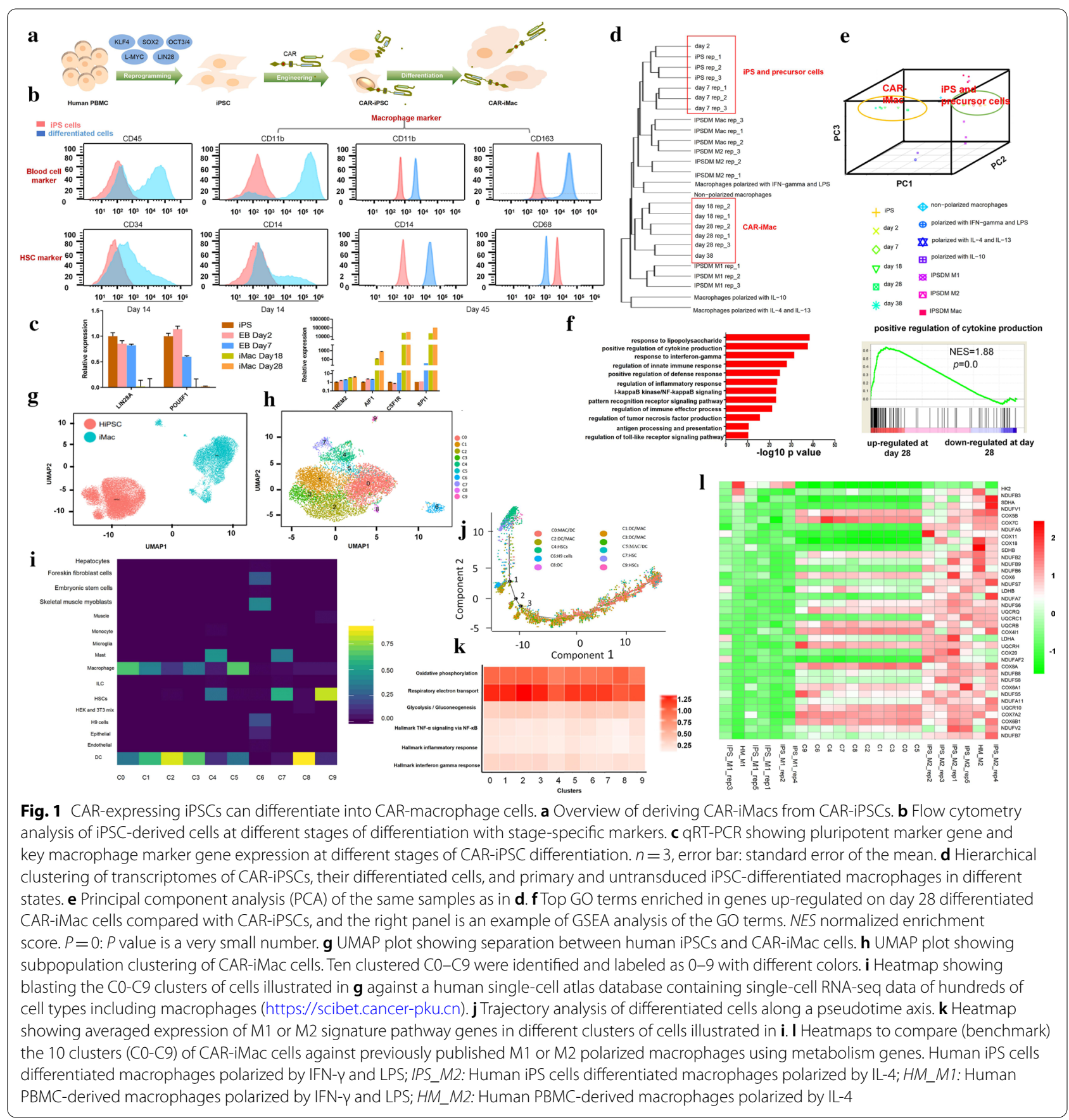


disappeared (Fig. 1c; Additional file 3: Table S1). RNAsequencing using differentiated cells showed that iPSCs clustered with precursor cells, and late-day differentiated cells clustered with primary macrophage cells, or untransduced iPSC-differentiated macrophage cells from previous studies [5, 6](Fig. 1d, e). GO analyses showed strong enrichment of innate immunity-related functions in 28-day differentiated cells (Fig. 1f; Additional file 4: Table S2).

Next, we further dissected their subpopulations by performing single-cell RNA-sequencing analysis. These cells clustered away from undifferentiated CAR-iPSCs (Fig. 1g), and they appeared to be largely homogenous with only a small number of cells not clustered with the main population (Fig. 1h). Blasting the differentiated single cells in a database of human cell atlas containing single-cell RNA-sequencing data revealed that these iMac cells mainly clustered with macrophages (Fig. 1i and Additional file 1: Fig. S6a). Trajectory analysis revealed that CAR-iMac cells went through a path from HSC to macrophage and DC cells without major branches (Fig. 1j, Additional file 1: Fig. S5c).

Moreover, all 10 clusters of differentiated cells showed strong signatures of M2 state [7-10] (Fig. 1k, Additional file 1: Fig. S6b). We further compared the single-cell data in the 10 clusters with bulk RNA-seq data from the LPS/ IFN- $\gamma$-polarized M1 cells or IL-4/IL-10-polarized M2 cells, by examining M1/M2-associated genes (Fig. 1l, Additional file 1: Fig. S5d), and found that most clusters were more similar to the M2 state particularly when using metabolism genes as markers [5, 6, 11-15].

Next, we incubated the CAR (CD19)-iMac cells, CAR (meso)-iMac cells, or control iMac cells with CD19expressing K562 leukemia cells or mesothelin-expressing OVCAR3/ASPC1 ovarian/pancreatic cancer cells.
Compared with K562 alone, K562-CD19 cells were more likely to be phagocytosed by CAR (CD19)-iMacs (Fig. 2a, b), and compared with control cells, CAR (meso)iMac showed increased phagocytosis activity against OVCAR3 and ASPC1 cells (Fig. 2g, h and Additional file 1: Fig. S7g). Intracellular signaling such as phosphorylation of ERK and NF- $\mathrm{kB}(\mathrm{P} 65)$ proteins were increased in CAR-iMacs co-cultured with CD19-expressing K562 cells compared to K562 cells, or to CAR-iMac cells cultured alone (Fig. 2c). We also examined cytokine gene expression in CAR(CD19)-iMac and CAR (meso)-iMac cells when they were incubated with tumor cells and found antigen-dependent increase in M1 pro-inflammatory cytokine expression(Fig. $2 \mathrm{~d}, \mathrm{j}$ and Additional file 1: Fig. S7h). Moreover, transcriptional analysis showed that CAR(CD19)-iMac cells and CAR(meso)-iMac cells showed strong enrichment of up-regulated genes in GO or KEGG terms of "positive regulation of cytokine secretion," "antigen processing and presentation," and "Tolllike receptor signaling pathway," indicating these cells are more wired toward the pro-inflammatory state, when they encounter the antigen (Fig. 2e, f, i and Additional file 1: Fig. S7i).

When injected into NSG mice, these CAR-iMac cells expanded in vivo till around day 3 for about two fold, and persisted till more than 20 days and gradually disappeared after around 30 days (Fig. $2 k$ ). To test their antitumor cell activity, we first intraperitoneally injected ovarian cancer cells HO8910 expressing a luciferase gene into NSG mice. In order to further polarize CARiMac cells toward M1, we treated them with IFN- $\gamma$ and washed IFN- $\gamma$ away before injection (Additional file 1: Fig. S8). CAR(meso)-iMac-treated mice showed reduced tumor burden compared to the control group on day 4 , 11, and 14 (Fig. 2l, m). These data demonstrate that the

\footnotetext{
(See figure on next page.)

Fig. 2 CAR-iMac cells showed antigen-dependent phagocytosis and anticancer cell functions in vitro and in vivo. a Confocal microscopy pictures showing phagocytosis of K562 or K562-CD19 cells (red) by CAR (CD19)-iMac cells (green). b Flow cytometry showing phagocytosis of K562 or K562-CD19 cells by CAR (CD19)-iMac cells. $\mathbf{c}$ Western blotting showing phosphorylation of ERK and NF-KB P65 in CAR (CD19)-iMac cells in the indicated conditions. $\mathbf{d}$ qRT-PCR showing cytokine gene mRNA expression when CAR (CD19)-iMac cells were incubated with K562 or K562-CD19 cancer cells for 24 h. $n=3$, error bar: standard error of the mean. e Top GO terms enriched in genes up-regulated in CAR-iMac cells. Right panel is GSEA analysis of "positive regulation of cytokine production." $\mathbf{f}$ Top KEGG pathways enriched in genes up-regulated in CAR-iMac cells. Right panel is GSEA analysis of "antigen processing and presentation." $\mathbf{g}$ Confocal microscopic images showing phagocytosis of OVCAR3 cells (red) by iMac or CAR (meso)-iMac cells (green). $\mathbf{h}$ Flow cytometry showing phagocytosis of OVCAR3 ovarian cancer cells by iMac or CAR (meso)-iMac cells. $\mathbf{i}$ GO term analysis with RNA-seq data showing the up-regulated genes in CAR (meso)-iMac cells. Right panel is GSEA analysis of "cytokine activity gene." $\mathbf{j}$ qRT-PCR showing cytokine gene mRNA expression when iMac or CAR (meso)-iMac cells were incubated with OVCAR3 cells for $24 \mathrm{~h}$. $n=3$. Error bar: standard error of the mean. $\mathbf{k} 3 \times 10^{6}$ DiR dye-labeled iMac cells were intraperitoneally injected into NSG mice. $n=3$. Error bars represent standard error of the mean. I $4 \times 10^{5}$ of luciferase-expressing ovarian cancer cells (HO8910) were intraperitoneally injected into NSG mice. Mice were treated $4 \mathrm{~h}$ later with I.P. injection of PBS, $4 \times 10^{6}$ iMac or $4 \times 10^{6} \mathrm{CAR}$ (meso)-iMac cells. Bioluminescence showing tumor development on the indicated days. Statistical analysis was calculated via one-way ANOVA with multiple comparisons between the PBS group and the CAR-iMac group. ${ }^{*} P<0.05$; ${ }^{*} P<0.01$. $\mathbf{m}$ Quantification of tumor burden (total flux) by bioluminescent imaging on day 4, 11, 14, and day 25 after CAR-iMac treatment was plotted. Data are presented as the median $\pm S D$, with statistical significance calculated via one-way ANOVA with multiple comparisons. ${ }^{*} P<0.05$; **P $<0.01$, ns not significant
} 

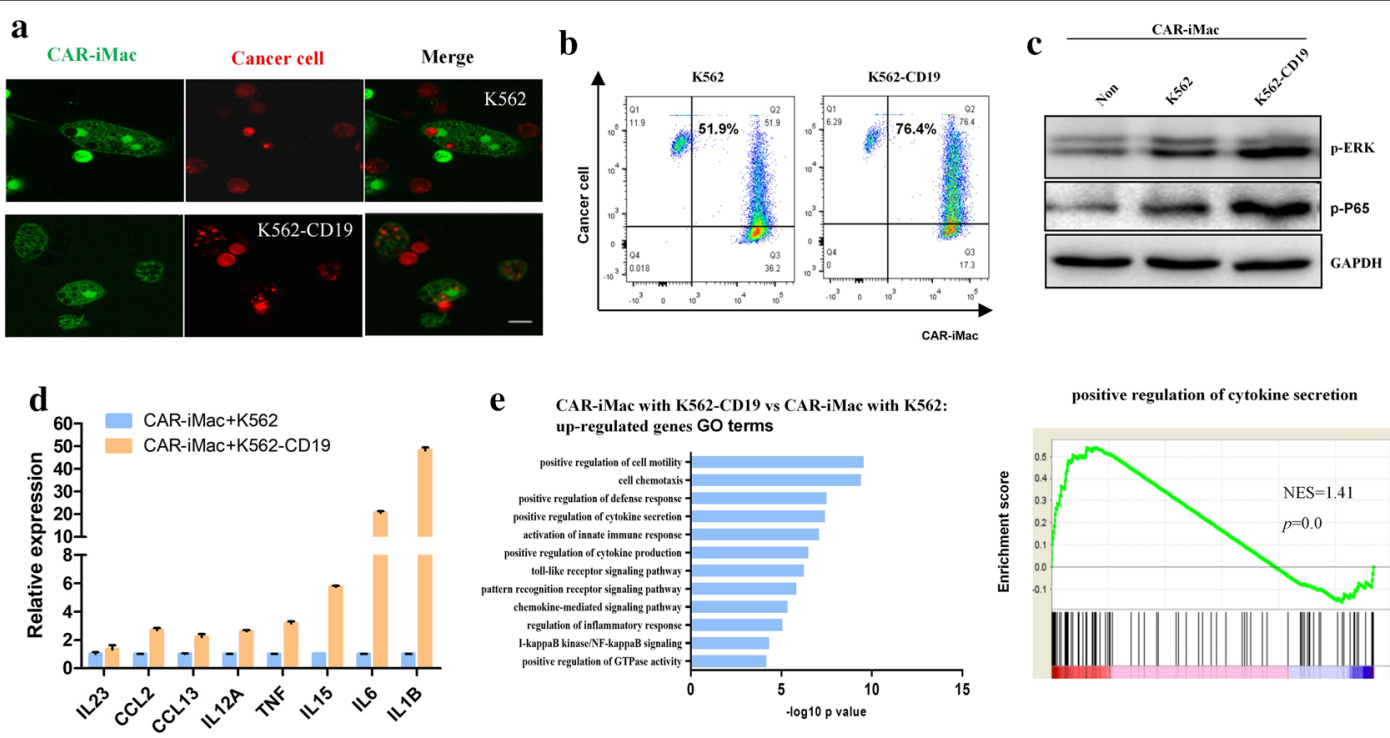

e CAR-iMac with K562-CD19 vs CAR-iMac with K562:

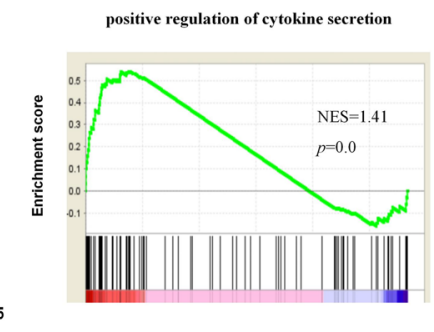

f

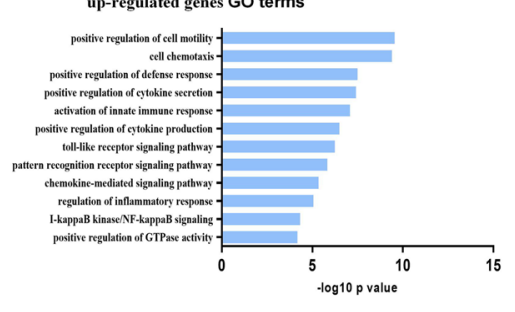

CAR-iMac with K562-CD19 vs CAR-iMac with K562 up-regulated genes KEGG terms

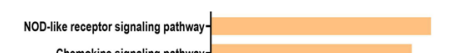
Chemokine signaling pathwayNF-kappa B signaling pathwayC-type lectin receptor signaling pathwayagocytos. rogamma R-mediated phagocytosisCytokine-cytokine recepter interaction-
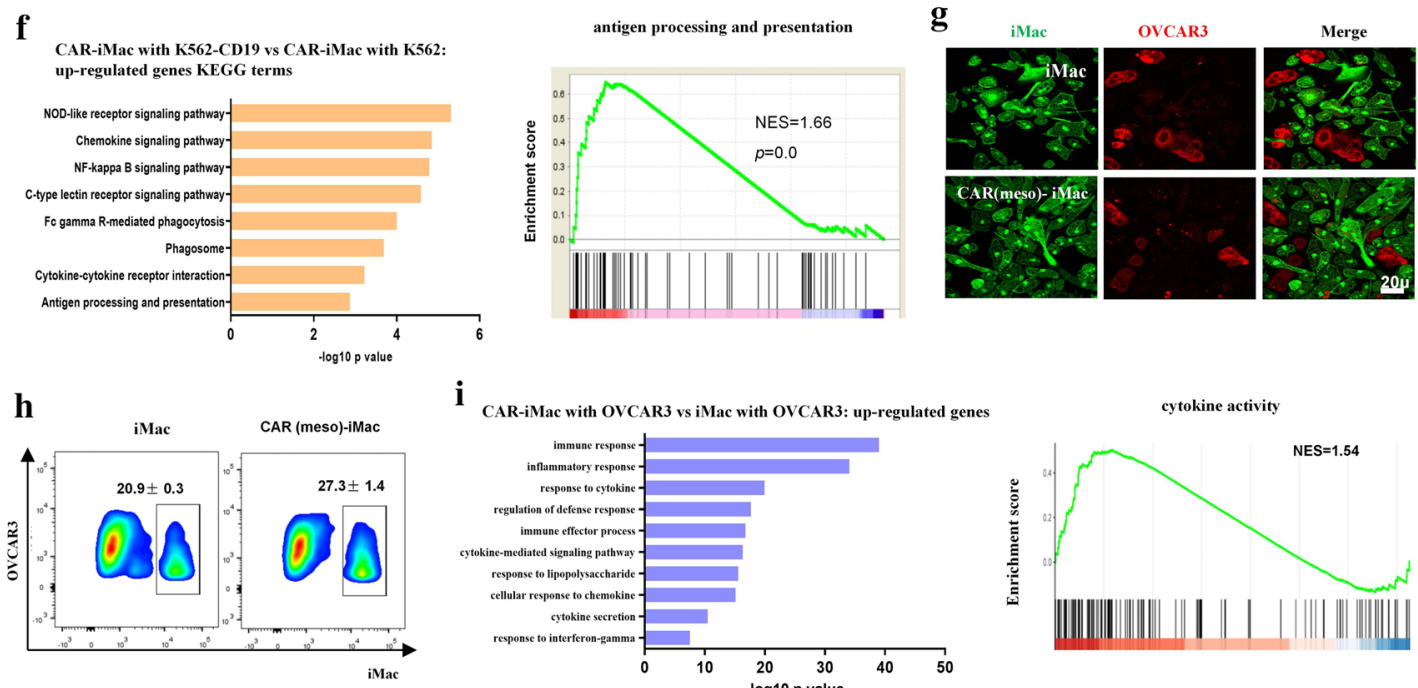

i

i CAR-iMac with OVCAR3 vs iMac with OVCAR3: up-regulated genes
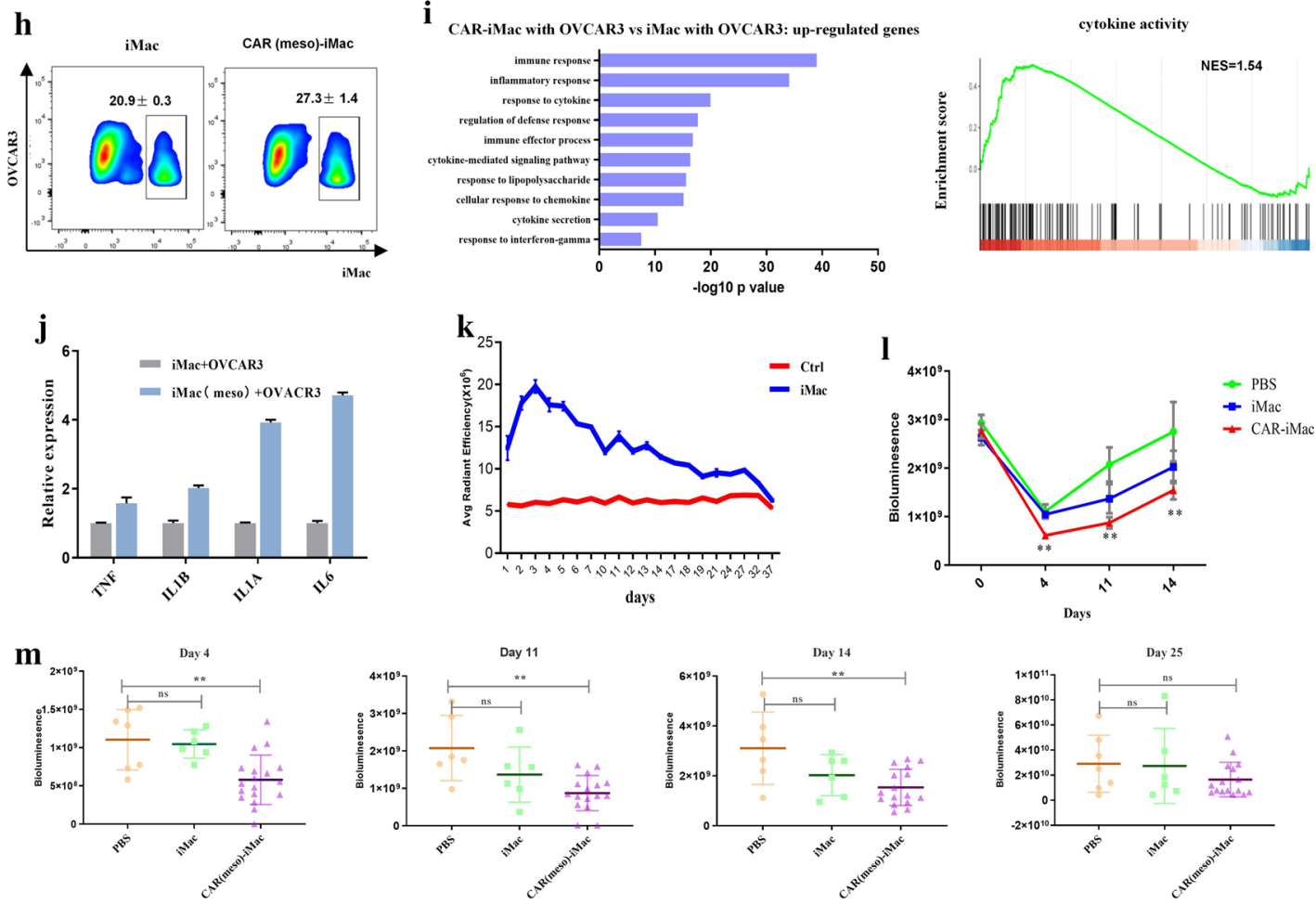
CAR confers anti-cancer cell activities in iMacs in vivo. The efficacy and persistency need to be further improved by designing more effective CARs or further modifying the CAR-iMac cells to stay constitutively active.

\section{Supplementary information}

Supplementary information accompanies this paper at https://doi. org/10.1186/s13045-020-00983-2.

Additional file 1. The main results of differentiation of CAR-expressing iPSCs into CAR-macrophage cells with antigen-dependent phagocytosis and anti-cancer cell functions in vitro and in vivo.

Additional file 2. Materials and Methods.

Additional file 3: Table S1. Primer sequences for qRT-PCR.

Additional file 3: Table S2. RNA-sequencing data analysis of iMac during the differentiation process from iPSCs.

\section{Abbreviations}

CAR: Chimera antigen receptor; DC: Dendritic cells; ERK: Extracellular-regulated protein kinase; HSC: Hematopoietic stem cell; ICT: Immune-checkpoint therapy; IFN-y: Interferon gamma; IP: Intraperitoneal injection; iPSC: Induced pluripotent stem cells; LPS: Lipopolysaccharide; MPS: Mononuclear phagocyte system; PBMC: Peripheral blood mononuclear cells; qRT-PCR: Quantitative reverse transcription $P C R$.

\section{Acknowledgements}

We thank George Q. Daley and Thorsten M. Schlaeger for providing reprogramming plasmids. We thank the Core Facilities of Zhejiang University School of Medicine for technical support and Center for Drug Safety Evaluation at Zhejiang University for animal technical support. We thank Ming Chen (College of Life Sciences, Zhejiang University) for computational analyses support and Harvey Lodish (MIT) for helpful Discussion. We thank CellOrigin for supporting this project.

\section{Authors' contributions}

$J Z, Q W, H H, Y X$, and $Y H$ designed and supervised the study. $L Z, L T, X D, J W, A L$, $M Z$, JX, WZ, YZ, ZS, HZ, and $Y W$ performed the experiments. HY performed the bioinformatics analysis. $\mathrm{HH}, \mathrm{HL}$, and GC provided key reagents and advice for the manuscript. All authors read and approved the final manuscript.

\section{Funding}

J.Z. is supported by the National Key Research and Development Program of China (2018YFA0107100, 2018YFA0107103, 2018YFC1005002), the National Natural Science Foundation projects of China $(31871453,91857116)$, the Zhejiang Innovation Team grant (2019R01004) and the Zhejiang Natural Science Foundation projects of China (LR19C120001).

\section{Availability of data and materials}

The datasets used and/or analyzed during the current study are available from the corresponding author on reasonable request.

\section{Ethics approval and consent to participate}

The animal study was performed in compliance with relevant regulatory standards. All animal studies are approved by ZJU IRB committee.

\section{Consent for publication}

Written informed consent for publication was obtained from all participants.

\section{Competing interests}

A patent has been filed related to the data presented. JZ and GC are founders and scientific advisors for CellOrigin.

\section{Author details}

${ }^{1}$ Center for Stem Cell and Regenerative Medicine, Department of Basic Medical Sciences, and The First Affiliated Hospital, Zhejiang University School of Medicine, Hangzhou, 310058, China. ${ }^{2}$ Institute of Hematology, Zhejiang University, Hangzhou 310058, China. ${ }^{3}$ Zhejiang Province Key Laboratory of Anti-Cancer Drug Research, Center for Drug Safety Evaluation and Research, Institute of Pharmacology and Toxicology, College of Pharmaceutical Sciences, Zhejiang University, Hangzhou 310058, China. ${ }^{4}$ The First Affiliated Hospital, Zhejiang University School of Medicine, Hangzhou 310000, China. ${ }^{5}$ Department of Neurology, The First Affiliated Hospital of Zhengzhou University, Zhengzhou 450052, China. ${ }^{6}$ Department of Genetics and Wyss Institute for Biologically Inspired Engineering, Harvard Medical School, Boston, MA 02115, USA. ${ }^{7}$ Zhejiang Laboratory for Systems and Precision Medicine, Zhejiang University Medical Center, 1369 West Wenyi Road, Hangzhou 311121, China.

Received: 20 October 2020 Accepted: 27 October 2020

Published online: 11 November 2020

\section{References}

1. Themeli M, Kloss CC, Ciriello G, Fedorov VD, Perna F, Gonen M, et al. Generation of tumor-targeted human $T$ lymphocytes from induced pluripotent stem cells for cancer therapy. Nat Biotechnol. 2013;31(10):928-33.

2. LiY, Hermanson DL, Moriarity BS, Kaufman DS. Human iPSC-derived natural killer cells engineered with chimeric antigen receptors enhance anti-tumor activity. Cell Stem Cell. 2018;23(2):181-92e5.

3. Morrissey MA, Williamson AP, Steinbach AM, Roberts EW, Kern N, Headley $\mathrm{MB}$, et al. Chimeric antigen receptors that trigger phagocytosis. Elife. 2018;7:e36688.

4. Klichinsky M, Ruella M, Shestova O, Lu XM, Best A, Zeeman M, et al. Human chimeric antigen receptor macrophages for cancer immunotherapy. Nat Biotechnol. 2020;38(8):947-53.

5. Zhang H, Xue C, Shah R, Bermingham K, Hinkle CC, Li W, et al. Functional analysis and transcriptomic profiling of iPSC-derived macrophages and their application in modeling Mendelian disease. Circ Res. 2015;117(1):17-28

6. Douvaras P, Sun B, Wang M, Kruglikov I, Lallos G, Zimmer M, et al. Directed differentiation of human pluripotent stem cells to microglia. Stem Cell Rep. 2017;8(6):1516-24

7. Sica A, Mantovani A. Macrophage plasticity and polarization: in vivo veritas. J Clin Investig. 2012;122(3):787-95.

8. Viola A, Munari F, Sanchez-Rodriguez R, Scolaro T, Castegna A. The metabolic signature of macrophage responses. Front Immunol. 2019;10:1462.

9. Galvan-Pena S, O'Neill LA. Metabolic reprograming in macrophage polarization. Front Immunol. 2014;5:420.

10. Kelly B, O'Neill LA. Metabolic reprogramming in macrophages and dendritic cells in innate immunity. Cell Res. 2015;25(7):771-84.

11. Mosser DM, Edwards JP. Exploring the full spectrum of macrophage activation. Nat Rev Immunol. 2008;8(12):958-69.

12. Xue J, Schmidt SV, Sander J, Draffehn A, Krebs W, Quester I, et al. Transcriptome-based network analysis reveals a spectrum model of human macrophage activation. Immunity. 2014;40(2):274-88.

13. Martinez FO, Gordon S. The M1 and M2 paradigm of macrophage activation: time for reassessment. F1000Prime Rep. 2014;6:13.

14. Martinez FO, Sica A, Mantovani A, Locati M. Macrophage activation and polarization. Front Biosci. 2008;13:453-61.

15. Lachmann N, Ackermann M, Frenzel E, Liebhaber S, Brennig S, Happle $C$, et al. Large-scale hematopoietic differentiation of human induced pluripotent stem cells provides granulocytes or macrophages for cell replacement therapies. Stem Cell Rep. 2015;4(2):282-96.

\section{Publisher's Note}

Springer Nature remains neutral with regard to jurisdictional claims in published maps and institutional affiliations. 